\title{
Access to Communication Channels and Use of Family Planning among Women in Tanzania: Spatial and Socio-demographic Analysis
}

\author{
Zuena Kilugwe ${ }^{1}$, Sosthenes Ruheza ${ }^{2}$ \\ ${ }^{1}$ Mzumbe University, School of Public Administration and Management, Tanzania \\ ${ }^{2}$ University of Iringa, Department of Community Development, Tanzania \\ *zkkilugwe@mzumbe.zc.tz,ruhezah@yahoo.com
}

\begin{abstract}
This study examined the relations and access to communication channels on the use of Family Planning (FP). Relationships between independent variables including access to communication channels and the use of FP services, and demographic characteristics were examined. This study used cross-sectional data from the Tanzania Demographic Health Survey and Malaria Indicator Survey (TDHS-MIS) of 2015-2016 from 11,127 women aged between 15-49 years. The sample was weighted to ensure representativeness. Univariate, bivariate and binary logistic regression analyses were used. Results in this study revealed a statically significant correlation between access to FP messages and use of family planning services ( $\mathrm{p}<.001)$. Access to radio, television, printed media, and mobile phones was observed more among women who were never in a union, those with tertiary education, women in urban areas and among those within a high wealth index. Use of FP services increased with the fluency of access to FP messages. Printed media predicted more likelihood in the use of FP at $\beta=0.460, \mathrm{p}<0.001$; radio at $\beta=0.368, \mathrm{p}<0.001$; health facilities at $\beta=0.284$, $\mathrm{p}<0.001$ and education level at $\beta=0.276, \mathrm{p}<0.001$. The study concludes that despite the fact that there was a correlation between access to FP messages and the use of FP, the use or not use of the same is also influenced by factors.
\end{abstract}

Keywords: Communication channels, family planning, maternal mortality, infant mortality, Tanzania.

\section{Background}

Fertility and projected population growth rates are significantly high in sub-Saharan Africa (World Health Organization, 2011 cited by Oseko, 2013). High population growth rate is a major international concern because it has the potential to hinder attainment of health and other development goals in the region (Cleland et al., 2006 cited by Nettey et al., 2015). In spite of the high fertility levels in most countries in sub-Saharan Africa, the potential economic benefits of Family Planning (FP) have not been fully realised in the sub-region (Nettey et al., 2015). FP has been promoted as a strategy to curb the problem of rapid population growth and its associated reproductive health problems (Ajaero, Odimegwu, \& Ajaero, 2016). FP reduces both maternal and infant mortality rates and it decreases abortions of undesired pregnancies, thus, increasing gender equality by enabling more girls to attain tertiary education, employment and increased income (Miller, 2011). A United Nations Population Fund, (2006) report indicates that, access to FP reduces global infant mortality at a rate of 2.7 million every year. In Kenya alone, it is estimated that FP is responsible for prevention of about 14,040 maternal deaths and 434,306 child deaths (Government of Kenya, 2007 cited by Oseko, 2013).

It has also been established that FP allows the spacing between births, hence, improving the health and wellbeing of a family; and of the population at large (Longwe, Huisman \& Smits, 2012; Westoff, 2012). Oseko (2013) observes that, when mediated by Interpersonal Communication (IPC) discussions of obtained messages prior to the decision to use FP, mass media may effectively motivate people to adopt FP. For example, various studies indicate that women, who were exposed to mass media messages and had discussed them with other people, were more likely to adopt FP compared to those who did not discuss the messages (Syed, Abdul \& Jan, 2008 cited by Oseko, 2013). Word-of-mouth was seen to be among the most effective

\footnotetext{
${ }^{1}$ : Mzumbe University, School of Public Administration and Management - (zkkilugwe@mzumbe.ac.tz - 
communication channels for acquiring knowledge and promoting desired changes in behaviour (Ajaero et al., 2016).

Different communication channels such as television, radios and mobile phones, printed media, health facilities and, health field workers have been globally used to communicate FP messages. Mass communication encouraged and motivated people to FP use. The common mass media that are used in disseminating FP messages are radio, television, cinema, printed media and traditional folk events (Kabir \& Islam, 2000 cited by Islam \& Hasan, 2016). The effectiveness of communication channels on influencing the use of FP varies considerably. For example, while mass media are very effective in the creation of awareness on FP, IPC moves an individual from being knowledgeable about FP to the uptake of the method and thereby acquiring new behaviour (International Planned Parenthood Federation, 2013). Both IPC and mass media messages avail women with awareness on FP use and the methods available. Contrary to the significance of the communication channels on influencing the use of FP, several authors such Apanga \& Adam (2015) and Hamid \& Stephenson (2006) observed that despite high awareness of FP services in the most communities, very few people were using the services. Socio-economic and demographic characteristics of the population potentially are responsible for this.

These include: level of education, place of residence, age, number of living children, and access to FP services. These form important determinants to use or not use of FP in sub-Saharan Africa (Kyalo, 1996 and Tuonane, 1999 cited by Oseko, 2013). A similar view was also reported by Davidson \& Jaccard (1979) cited by Islam \& Hasan (2016) factors such as age, son preferences, a number of sons and daughters also influenced the couple's use of FP. In Bangladesh, factors such as administrative areas, educational level, health workers visit, religion, region, current children, age, and occupation were found to influence married women's uptake of FP services (Rahman et al., 2011 cited by Islam \& Hasan, 2016). It is against this background that this study, using the Tanzania Demographic Health Survey and Malaria Indicator Survey (TDHS-MIS) data of 2015-16, examined the significance of access to different communication channels on FP messages on the use of the service among women in Tanzania. It is envisaged that the findings will be useful in determining the most effective communication channels for conveying FP messages. This may contribute to the reduction of maternal and infant mortality rates, reduce socio-economic problems associated with rapid population growth, reduce abortions of unwanted pregnancies and increase gender equality.

\section{Literature Review}

Socio-Demographic Characteristics, Access to Communication Channels and FP Use: Family Planning refers to the control of a number of children a woman can give birth to (Oseko, 2013). It is a process of avoiding too early, too close, too many and too late childbearing. FP methods the use of condoms, oral contraceptives, implants, injections, Intrauterine Contraceptive Devices IUCD) and female sterilization (hysterectomy), cervical cap, coitus interrupt Lactation Amenorrhea Method (LAM) among others (Oseko, 2013). FP involves behaviour change and this can be achieved through Information, Education and Communication (IEC) advocacy campaigns in the society, especially among women. Access to appropriate knowledge and information about the new behaviour and its relevance, people are able to make informed choices about their reproductive health (Oseko, 2013). Communication is an essential element in reproductive health, as it motivates women to change from unhealthy behaviour and practice healthy behaviour all through. In FP, Behaviours Change Communication (BCC) help people practice healthy behaviour by increasing awareness of reproductive health, improve women's health status and related longterm outcomes. It also motivates individuals to seek services and help them to successfully use their FP method of choice (Oseko, 2013). Results indicated that exposure to BCC messages was associated with higher FP intentions and use (Gupta, Katende \& Bessinger, 2003). There is evidence from a number of studies that individuals' exposure to mass media messages promoting FP influences contraceptive behaviour (Piotrow et al., 1990, Bankole; Rodriquez \& Westoff, 1996, Westoff \& Bankole, 1997 and Kincaid, 2000 cited by Gupta et al., 2003). For example, in Nigeria, the use of modern FP, intent to use, and desire for fewer children were found to be associated with exposure to media messages on FP (Bankole et al., 1996 cited by Gupta et al., 2003). A similar study in Tanzania found that women exposed to a mix of media promoting FP services were 
more likely to use FP than those who did not have access to messages on FP (Jato et al., 1999 cited by Gupta et al., 2003).

This suggests that different messages and approaches in BCC are required to reach people at different stages in the communication process (Gupta et al., 2003). Communication through mass media has been popular due to the opportunity it affords to reach a large audience and address issues that are sensitive or culturally taboo in an entertaining and informative manner and, are most effective when combined with other intervention components such as social marketing or IPC interventions (Mwaikambo et al., 2011 cited by Grant \& Bhardwaj, 2016). For example, a study in Nepal, revealed that exposure to mass media had an indirect effect on FP use through an increase in IPC, as well as positive changes in attitudes and perceived social norms regarding FP (Storey et al., 1999 cited by Gupta et al., 2003). Similarly, women exposed to a mass media campaign in Tanzania were found to have more positive attitudes towards FP and facilitates discussion on FP issues with their spouses (Jato et al., 1999 cited by Gupta, 2003). Furthermore, a study in Tanzania by Jato et al. (1999) and in Mali by Kane et al. (1998) cited by Gupta et al. (2003) found that the more types of media sources of FP messages, the greater the likelihood of FP use. IPC is a very effective and efficient in health communication as it helps to influence, discover and share information among individuals involved as it permits dialogue and immediate response to the individual (Oseko, 2013).

IPC approaches can include one-on-one discussions, small-group sessions, and facilitator-led curriculumbased programmes (Gupta et al., 2003). IPC helps in the effective delivery of information to community members and move individuals from being knowledgeable about FP to uptake methods and continued practices of new behaviour (International Planned Parenthood Federation, 2013). IPC supported by mass media could play a key role in increasing correct knowledge on FP, addressing misconceptions and triggering spousal communication (Khan et al., 2013 cited by Grant \& Bhardwaj, 2016). Apart from access to FP messages, studies show that several socio-demographic factors are associated with not only to the access to FP messages but also to the change in attitude and practices about the use of FP. Several studies such as that in Senegal and Burkina Faso by Jacobs (2016), observe that FP messages via mass media were not reaching the poor, less-educated, and rural women; access to printed media increased with women's levels of education (Amin, 2014; Jacobs, 2016). Moreover, the study by Jacobs (2016) revealed lower levels of exposure among adolescent women across all forms of media.

Socio-demographic factors often cited in the literature as important determinants of changing FP attitudes and practices include age, parity, the number of children ever born and, place of residence have repeatedly been found to influence reproductive behaviours (Gupta et al., 2003). For example, a study in Uganda on FP prevalence among women ranged from $6 \%$ for those with no formal education to $36 \%$ for those with at least some secondary schooling, with only $35 \%$ of women with no education reported intent to use FP in the near future, compared with those in the highest educational category $47 \%$ (Gupta et al., 2003). Women's exposure to BCC messages was found to be significantly associated with increased FP use, all else being equal. Not only were women with no exposure less likely to be using FP compared to their counterparts who were exposed to anyone message type, but also greater likelihood of FP use with exposure through multiple media channels showed evidence of a dose-response effect. At the same time, FP prevalence was found to have increased significantly over time (Gupta et al., 2003).

\section{Methods}

Setting: The United Republic of Tanzania is a multicultural and multi-religion country in East Africa. The country has a mixed economy. In 2016, the population of Tanzania mainland was projected at 50.1 million (National Bureau of Statistics, 2015); with a total fertility rate of 5.2 (Tanzania Demographic Health Survey and Malaria Indicator Survey, 2015-2016). The high population growth rate in Tanzania has been brought about by high fertility and declining mortality levels. The population of Tanzania has continued to be predominantly rural despite the increase in the proportion of urban residents over time, from 6\% in 1967 to 30\% in 2012 (National Bureau of Statistics, 2015). 
Source of Data: This research used cross-sectional data from the Tanzania Demographic Health Survey and Malaria Indicator Survey (TDHS-MIS) of 2015-2016 from 11,127 women aged between 15-49 years. The survey collected information on fertility levels, marriage, sexual activity, fertility preferences, awareness and use of FP methods, breastfeeding practices, nutrition, childhood and maternal mortality, maternal and child health, malaria, and other health-related issues. In addition, the Tanzania Demographic Health Survey and Malaria Indicator Survey 2015-16 provided estimates of anaemia prevalence among children age 6-59 months and women age 15-49 years, estimates of malaria prevalence among children age 6-59 months, and estimates of iodine concentration in household salt and women's urine. The sample for the survey was derived from the use of a two-stage cluster sampling design. In stage one, 608 Enumeration Areas (EAs) were selected as clusters from a sampling frame developed for the 2012 Tanzania Population and Housing Survey. From the selected EAs, 22 households were systematically selected in stage two and all women aged 15-49 years who were present in the households, including visitors who spent the preceding night in the household, were included in the analysis.

Variables Used for the Study: The study used two major variables for analysis: using or intend to use FP (both modern and traditional methods) (dependent variable) and access to FP messages (independent dummy variable) and spatial and socio-demographic characteristics of the population. Dependent variables included women aged 15-49 years who were using or intended to use both modern and traditional FP. This variable was measured as a dichotomous variable coded as the use of FP or no use or no intention to use FP. The independent variable was access to FP messages via radio, television, printed media, mobile phones, health facilities and field health workers. The variable was also measured dichotomously, and was coded as having or not having access to FP messages via different communication channels.

Data Analysis: The Statistical Package for Social Sciences (SPSS) version 16 was used for data analysis. Descriptive and inferential data analyses were done. The descriptive analysis involved univariate analysis of the socio-economic and demographic characteristics of the population, while bivariate analysis between the use of FP and each of the methods of conveying FP messages (radio, television, mobile phone, printed media, and health facility and health field worker). Finally, logistic regression was used to estimate the effectiveness of each of the communication channels and socio-economic and demographic characteristics of the population on the use of FP among women in Tanzania.

\section{Results and Discussion}

Frequency of Access to FP Messages and the Use of FP: Results in Table 1 show that there was a statistically significant relationship $(\mathrm{p}<0.001)$ between the frequency of exposure to FP messages and the use of FP. The percentage of the women who used FP increased with the fluency of exposure to FP messages from $64.5 \%$ (1318) for those who had never received FP messages to $73.1 \%$ (3101) among those who occasionally received the messages and, almost 80\% (1330) of the women who received FP messages regularly.

Table 1: Cross-tabulation between Frequency of Access to FP Message and the use of FP $(\mathrm{P}<.000)$

\begin{tabular}{llll}
\hline & \multicolumn{3}{l}{ Frequency of access to FP message } \\
use/or not use of FP & Not at all & Occasionally & Daily \\
\hline Not using/not intend to use & $35.8 \%(735)$ & $26.9 \%(1140)$ & $20.1 \%(335)$ \\
Use FP & $64.5 \%(1318)$ & $73.1(3101)$ & $79.9 \%(1330)$ \\
Total & $100 \%(2053)$ & $100 \%(4241)$ & $100 \%(1665)$ \\
\hline
\end{tabular}

Personal Characteristics of the Respondents and Access to FP Messages: Results in Table 2 indicate that there is a significant correlation between independent variables and access to FP messages via radios $(\mathrm{p}<0.001)$. Almost three-fifths $(60.0 \%)$ of the women in the age groups $20-24,25-29$ and $30-34$ had the highest access to FP messages via radio, while those in the age group $45-49$ had $50 \%$ of accessing FP messages via radio. Women who were not in the union had the highest access to FP messages via radio $67 \%$ (334), followed by divorced almost 63\% (603), the married 58\% c (4986) and the widowed 58\% (95). Results further revealed that access to FP messages via radio increased with women's education level. The findings indicated that only $42 \%$ (918) of the women with no formal education had access to FP messages. 
Whereas 63\% (3866) were those with primary education, almost 66\% (1166) and, 76.4\% (68) of women were those with higher levels of education. Results further indicated that 54.5\% (4270) of the rural residents had access to FP message via radio, whereas, $73.1 \%$ (1748) included those among the urban dwellers. Access to FP messages via radio increased with the increased wealth status, as only $50.1 \%(2220)$ of the women with a low wealth index had access to FP messages via radio compared to 67.1\% (2577) of the women within a high wealth index. Results (Table 2) indicate that there was a statistically significant correlation between independent variables and access to FP messages via television ( $\mathrm{p}<0.001)$. Nearly $25 \%$ ) of women within the age groups 20-24, 25-29 and 30-34 accessed FP messages via television, while, 18\% (348) accounted for those in the age group 15-19 and 13\% (465) in the age group 40-44. Women who were not in the union had the highest access to FP messages via television. These accounted to41.4\% (206) compared to $26.1 \%$ (43) of the widowed, $25.5 \%$ (245) of the divorced and almost $21 \%$ (1791) of the married women. Results further revealed that access to FP messages via television increased with the education level of the women. The findings indicate that only 6\% (129) of the women with no formal education had access to FP message via television compared to $72 \%$ (64) of the respondent with a higher education level. Place of residence was another important factor that determines women's access to FP messages. While $14.1 \%$ (1103) of the women residing in rural areas had access to FP messages via television, urban dwellers accounted for 49.4\% (1182). Similarly, only 8.3\% (369) of the women in the low wealth index had access to FP messages via television compared to $43.2 \%$ (1647) of those in the high wealth index.

Results further show that there was a statistically significant association $(\mathrm{p}<0.001)$ between access to FP messages via printed media and independent variables. For instance, women within an age group 20-24 had $23.1 \%$ (563) of access, whereas, those in age groups 30-34 and $25-29$ had $21.4 \%$ (420) and $20.1 \%$ (510) of access, respectively. Those in age group $45-49$ had 7.4\% (40) access. Results also revealed that women who were not in the union had 37\% (185) access to FP messages via printed media, the widowed had $24.2 \%$ (40), the divorced $22.1 \%$ (213), whereas, married women had 18\%(1526) access through the media. As regards education, results revealed that $1.6 \%$ (35) of those with no formal education, $22 \%$ (1338) with primary education, $30.4 \%$ (539) with secondary education, and 58.4\% (52) with tertiary education had access to FP messages via print media. Women in urban areas had $32.4 \%$ (775) access to PF messages via print media compared to $15.2 \%$ (544) of their rural counterparts. The effects of wealth were also analysed in terms of women's access to FP messages through print media. Results indicated that women within the low wealth index had $12.3 \%$ (544) access, whereas, those within the middle and high wealth indices had 16.7\% (333) and $28.5 \%$ (1087) of access, respectively. Results also indicated that there was a statistically significant correlation between independent variables and access to FP messages via mobile phones $(\mathrm{p}<0.001)$. For instance, access to FP messages via mobile phones decreased with increased age. For instance, women in the age group 25-29 had almost $5 \%$ (119) access to FP messages, those in the age group 20-24 and 30-34 had $4.1 \%$ (99), whereas, the lowest percent was $0.8 \%$ (2) among those in the age group 45-49.

Findings also indicated that access to FP messages via mobile phones increased with women's education level. Almost 35\% (31) of women with the highest level of education had access to FP messages via mobile phones compared to only $0.5 \%$ (11) of the women with no formal education. Results further showed that, while 9\% (216) in urban residences had access to FP messages via mobile phones, only 2\% (154) of rural residents had access to FP messages via this medium. Similarly, the difference was observed based on the wealth index. About 8\% (301) of the women in the high and 1\% (39) in the low wealth index had access to FP messages via mobile phones. Marital status had a significant correlation with access to FP messages via health facilities $(p<0.001)$. Access to FP messages via health facilities was reported in 46\% (3032) of married women, $42.3 \%$ (159) among those who were never in a union, 39\% (284) among the divorced, whereas, the percentage of the widowed was only $29.4 \%$ (32). Findings also indicated that education was an important factor that influenced access to FP messages. Findings showed that $46.2 \%$ (2207) of the women with primary education, $42.1 \%$ (587) with secondary, $46 \%$ (33) with tertiary education and $43.1 \%$ (680) of those with no formal education had access to FP messages via health facilities. Similarly, about 45.6\% (2700) of women in the rural areas accessed FP messages via health facilities compared to 43\% (807) of the urban residents. Access to FP messages decreased with increased wealth index among women; almost 47\% (1570) included women in the low wealth index while $42 \%$ (1245) included those in the high wealth index. 
Furthermore, results (Table 2) show that there was no significant correlation between independent variables and access to FP messages via field workers. Results show that women in age group 15-19 had 34.6\% (8), 2024 had almost 48\% (43), 25-29 had 57\% (65), and 30-34 had 36.4\% (24) access to FP messages via field workers. Those in the age groups $40-44$ and $45-49$ had 50\% (16) and $80 \%$ (8) respectively. Results also revealed that access to FP messages via field workers varied with marital status. Access via field workers was reported among $46 \%$ (11) of the women who were never in a union, $47.5 \%$ (171) of the married, 44.4\% (4) of the widowed and 51.5\% (17) of the divorced women. Similarly, it was also found that $50.6 \%$ (39) of the women who had no formal education, $45.2 \%$ (113) with primary, with secondary education with tertiary education had access to FP messages via field workers. The difference, however, was not significant ( $\mathrm{p}=0.681$ ). Furthermore, results indicated that 48\% (145) of women who resided in rural areas accessed FP messages via field workers. Those in the urban included 47.5\% (53) of women. The difference, however, was not statically significant $(\mathrm{p}=0.977)$. Likewise, access to FP messages via field workers varied considerably with the women's wealth index. This study showed that $50.3 \%$ (76) of the women in the low wealth index accessed FP messages via field workers, whereas, 49.4\% (40) and $45 \%$ (87) included those within the middle and high wealth indices, respectively. The difference was not statistically significant $(p<0.564)$.

Binary Logistic Regression between the Dependent and Independent Variables: Logistic regression was run on the study findings. Results from the regression model (Table 4) indicated that the model's was significant $(\mathrm{p}<0.001)$, with Wald value $=410.726$ and 10 degree of freedom. We, hence, concluded that the model is statistically significant to predict how independent variables affect the dependent variable in this study. Furthermore, the model, as reflected, indicates that of all the independent variables: television and health facilities, age groups, and education level were statistically significant at $p<0.001$, while printed media were statistically significant at $\mathrm{p}<0.001$. Mobile phones, radios, marital status and wealth index were not statistically significant.

Access to Different Sources of FP Messages and the Use of FP: Results (Table 3) indicated that 80.5\% (2840) of the women who were using FP accessed FP message via radio $(\mathrm{p}<0.001), 84.8 \%$ (1937) via television $(\mathrm{p}<0.001), 87.5 \%$ (1718) via printed media( $\mathrm{p}<0.001), 89.2 \%(7354)$ via mobile phones $(\mathrm{p}<0.001)$, $85.2 \%(173)$ via field workers $(\mathrm{p}<0.163)$ and $80.2 \%(2814)$ via health facilities $(\mathrm{p}<0.001)$. Results also revealed that radio, television, printed media, and mobile phones were most accessed by women who were never in a union, those with tertiary education, women in urban areas and by those within the high wealth index. Health facilities were mostly accessed by married women; those with primary and tertiary education; those in rural areas and those within the low wealth index. Field workers were mostly accessed by married women, women in the old age, those with tertiary education, women in rural areas and those within the low wealth index. Results further indicate that women in the reproductive age have the highest access to the sources of FP messages, except via field workers that were most accessed mostly by women aged between 45-49. Consistent with these study findings, the study in Senegal and Burkina Faso by Jacobs (2016) observes that FP messages via mass media are not reaching the poor, less-educated, and rural women; access to printed media increased with women's levels of education (Amin, 2014; Jacobs, 2016).

The contrasting finding is that by Jacobs (2016) who observed lower levels of exposure among adolescent women across all forms of media. Arguably, effectiveness and efficiency of communication channels on FP messages significantly differ within and between countries; women's exposure to FP messages via multiple forms of media as compared to one was more likely to influence their intention to use FP (Oseko, 2013). Contrary to the study finding, the study in Pakistan observed that despite the high awareness of FP services in the community, very few people use the service (Hamid \& Stephenson, 2006; Apanga \& Adam, 2015; Ochako, et al., 2015). It is worth noting that women's decisions to use FP also depended on other factors such as age, son preferences and a number of sons and daughter (Davidson \& Jaccard, 1979 cited by Islam \& Hasan, 2016). For example, according to Ajaero et al. (2016), in most cases, people in rural areas do not adopt modern FP as they prefer large family size to meet man labour required for production. Moreover, the study findings indicate women who use FP haven ever accessed FP messages from formal communication media. This may suggest existence of informal messages on FP that might have influenced them. 
Journal of Social and Development Sciences (ISSN 2221-1152)

Vol. 9, No. 3, pp. 55-65, September 2018

\begin{tabular}{|c|c|c|c|c|c|c|c|c|c|c|c|c|c|c|c|c|c|c|}
\hline \multirow[t]{2}{*}{$\begin{array}{l}\text { Population } \\
\text { characteristics }\end{array}$} & \multicolumn{3}{|l|}{ Radio } & \multicolumn{3}{|c|}{ Television } & \multicolumn{3}{|l|}{$\begin{array}{l}\mathrm{NP} / \mathrm{M} \\
\mathrm{Z}\end{array}$} & \multicolumn{3}{|c|}{$\begin{array}{l}\text { Mobile } \\
\text { Phone }\end{array}$} & \multicolumn{3}{|c|}{$\begin{array}{l}\text { Health } \\
\text { Facilities }\end{array}$} & \multicolumn{3}{|c|}{$\begin{array}{l}\text { Field } \\
\text { Workers }\end{array}$} \\
\hline & Yes & $\%$ & Sig & Yes & $\%$ & Sig & Yes & $\%$ & Sig & Yes & $\%$ & Sig & Yes & $\%$ & Sig & Yes & $\%$ & Sig. \\
\hline \multicolumn{19}{|l|}{ Age group } \\
\hline $15-19$ & 348 & 54.7 & & 115 & 18.1 & & 106 & 16.7 & & 11 & 1.7 & & 208 & 42 & & 8 & 34.6 & \\
\hline $20-24$ & 1463 & 60.2 & & 609 & 25 & & 563 & 13.1 & & 99 & 4.1 & & 852 & 45.7 & & 43 & 47.8 & \\
\hline $25-29$ & 1548 & 60.9 & & 633 & 24.9 & & 510 & 20.1 & & 119 & 4.7 & & 923 & 46.8 & & 65 & 57 & \\
\hline $30-34$ & 1184 & 60.2 & & 472 & 24 & & 420 & 21.4 & & 81 & 4.1 & & 638 & 42.3 & & 39 & 42.4 & \\
\hline $35-39$ & 88.1 & 57.1 & & 306 & 19.8 & & 248 & 16.1 & & 43 & 2.8 & & 557 & 46.5 & & 24 & 36.4 & \\
\hline $40-44$ & 465 & 54.5 & & 114 & 13.4 & & 98 & 11.5 & & 15 & 1.8 & & 259 & 42.2 & & 16 & 50 & \\
\hline $45-49$ & 129 & 50 & 0.000 & 36 & 14 & 0.000 & 19 & 7.4 & 0.000 & 2 & 2.8 & 0.000 & 70 & 42.4 & 0.433 & 8 & 80 & 0.082 \\
\hline \multicolumn{19}{|l|}{ Marital status } \\
\hline Never in union & 334 & 67.1 & & 206 & 41.4 & & 185 & 37.1 & & 28 & 5.6 & & 159 & 42.3 & & 11 & 45.8 & \\
\hline Married & 4986 & 57.9 & & 1791 & 20.8 & & 1526 & 17.7 & & 297 & 3.5 & & 3032 & 45.9 & & 171 & 47.5 & \\
\hline Widowed & 95 & 57.6 & & 43 & 26.1 & & 40 & 24.2 & & 10 & 6.1 & & 32 & 29.4 & & 4 & 44.4 & \\
\hline $\begin{array}{l}\text { Divorced } \\
\text { Education } \\
\text { level }\end{array}$ & 603 & 62.7 & 0.000 & 245 & 25.5 & 0.000 & 213 & 22.1 & 0.000 & 35 & 3.6 & 0.026 & 284 & 39 & 0.000 & 17 & 51.5 & 0.966 \\
\hline No formal & 918 & 41.7 & & 129 & 5.9 & & 35 & 1.6 & & 11 & 0.5 & & 680 & 43.1 & & 39 & 5.6 & \\
\hline Primary & 3866 & 62.7 & & 1309 & 21.2 & & 1338 & 21.7 & & 162 & 2.6 & & 2207 & 46.2 & & 113 & 45.2 & \\
\hline Secondary & 1166 & 65.7 & & 783 & 44.1 & & 539 & 30.4 & & 166 & 9.4 & & 587 & 43.1 & & 44 & 51.2 & \\
\hline $\begin{array}{l}\text { Higher } \\
\text { Place } \\
\text { residence }\end{array}$ & 68 & 76.4 & 0.000 & 64 & 71.9 & 0.000 & 52 & 58.4 & 0.000 & 31 & 34.8 & 0.000 & 33 & 45.8 & 0.021 & 7 & 53.8 & 0.681 \\
\hline Rural & 4270 & 54.5 & & 1103 & 14.1 & & 1189 & 15.2 & & 154 & 2 & & 2700 & 45.6 & & 145 & 47.7 & \\
\hline Urban & 1748 & 73.1 & 0.000 & 1182 & 49.4 & 0.000 & 775 & 32.4 & 0.000 & 216 & 9 & 0.000 & 807 & 42.6 & 0.021 & 53 & 4.5 & 0.977 \\
\hline \multicolumn{19}{|l|}{ Wealth index } \\
\hline Poor & 2220 & 50.1 & & 369 & 8.3 & & 544 & 12.3 & & 39 & 0.9 & & 1570 & 46.9 & & 76 & 50.3 & \\
\hline Middle & 1221 & 61.4 & & 269 & 13.5 & & 333 & 16.7 & & 30 & 1.5 & & 692 & 46.5 & & 40 & 49.4 & \\
\hline Rich & 2577 & 67.5 & 0.000 & 1647 & 43.2 & 0.000 & 1087 & 28.5 & 0.000 & 301 & 7.9 & 0.000 & 1245 & 41.7 & 0.000 & 87 & 44.8 & 0.564 \\
\hline
\end{tabular}




\begin{tabular}{|c|c|c|c|c|}
\hline \multicolumn{5}{|c|}{$\begin{array}{l}\text { Journal of Social and Development Sciences (ISSN 2221-1152) } \\
\text { Vol. 9, No. 3, pp. 55-65, September } 2018\end{array}$} \\
\hline \multicolumn{5}{|c|}{$\begin{array}{l}\text { Table 3: Bivariate and Chi-Square Analysis of Access to Different Sources of FP Messages nd Use of } \\
\text { FP/Not Using FP }\end{array}$} \\
\hline Source of FP Messages & Status & Yes & No & p-value \\
\hline \multirow{7}{*}{ Radio message } & & $19.50 \%$ & $32.60 \%$ & \multirow{7}{*}{0.000} \\
\hline & NUFP & $(1174)$ & $(1375)$ & \\
\hline & & $80.50 \%$ & $67.40 \%$ & \\
\hline & UFP & $(4844)$ & $(2840)$ & \\
\hline & Total & $100 \%$ (6018) & $100 \%(4215)$ & \\
\hline & NUFP & $15.2 \%(343)$ & $\begin{array}{l}27.70 \% \\
(2201)\end{array}$ & \\
\hline & UFP & $84.8 \%$ (1937) & $\begin{array}{l}72.30 \% \\
(5747)\end{array}$ & \\
\hline \multirow[t]{3}{*}{ Television message } & Total & $100 \%(2285)$ & $100 \%(7948)$ & \multirow[t]{3}{*}{0.000} \\
\hline & NUFP & $12.50 \%(246)$ & $\begin{array}{l}27.90 \% \\
(2303)\end{array}$ & \\
\hline & UFP & $\begin{array}{l}87.50 \% \\
(1718)\end{array}$ & $\begin{array}{l}72.10 \% \\
(5966)\end{array}$ & \\
\hline \multirow[t]{3}{*}{ Newspaper/magazine } & Total & $100 \%(1964)$ & $100 \%$ (8269) & \multirow[t]{3}{*}{0.000} \\
\hline & NUFP & $10.80 \%(40)$ & $\begin{array}{l}25.40 \% \\
(2509)\end{array}$ & \\
\hline & UFP & $\begin{array}{l}89.20 \% \\
(7354)\end{array}$ & $\begin{array}{l}74.60 \% \\
(7354)\end{array}$ & \\
\hline \multirow[t]{3}{*}{ Mobile phone message } & Total & $100 \%(7394)$ & $100 \%(9863)$ & \multirow[t]{3}{*}{0.000} \\
\hline & NUFP & $14.80 \%(30)$ & $10.30 \%(23)$ & \\
\hline & UFP & $85.20 \%(173)$ & $89.70 \%(200)$ & \\
\hline \multirow[t]{2}{*}{ Field workers' } & Total & $100 \%(203)$ & $100 \%(223)$ & \multirow[t]{2}{*}{0.0163} \\
\hline & $\begin{array}{l}\text { NUFP } \\
\text { UFP }\end{array}$ & $\begin{array}{l}19.80 \% \\
(693) \\
80.20 \% \\
(2814)\end{array}$ & $\begin{array}{l}25.70 \% \\
(1107) \\
74.30 \% \\
(3205)\end{array}$ & \\
\hline Health Facilities & Total & $100 \%(3507)$ & $100 \%(4312)$ & 0.000 \\
\hline
\end{tabular}

NUFP= not use Family Planning; UFP= use Family Planning, FP= Family Planning

Table 4: Binary Logistic Regression Results of the Predictors of the Current Use of FP and Access to FP Messages

\begin{tabular}{llllll}
\hline Independent Variables & $\boldsymbol{\beta}$ & SE & Wald & D & p-value \\
\hline Constant & & & $\chi^{2}$ & & \\
Radio & 1.537 & 0.219 & 49.64 & & 0.000 \\
Television & 0.368 & 0.059 & 37.233 & 1 & 0.050 \\
Newspaper/magazine & 0.168 & 0.086 & 3.843 & 1 & 0.000 \\
Mobile phone & 0.460 & 0.00 & 26.295 & 1 & 0.010 \\
Health facilities & 0.392 & 0.205 & 3.656 & 1 & 0.056 \\
Age & 0.284 & 0.057 & 25.128 & 1 & 0.000 \\
& -0035 & 0.004 & 82.994 & 1 & 0.000
\end{tabular}




\begin{tabular}{llllll} 
Residence & -0.065 & 0.08 & 0.654 & 1 & 0.419 \\
Education level & 0.276 & 0.049 & 32.44 & 1 & 0.000 \\
Wealth index & 0.036 & 0.025 & 2.112 & 1 & 0.146 \\
Marital status & 0.016 & 0.03 & 49.064 & 1 & 0.609 \\
\hline
\end{tabular}

DF: 10 P. $<.05 ; \mathrm{R}^{2}=0.078$

The Model Was: UFP $=0.490+0.448 \mathrm{X} 1-0.253 \mathrm{X} 2+0.200 \mathrm{X} 3+0.124 \mathrm{X} 4+\varepsilon$; where UFP $=$ Use of FP, $\mathrm{X} 1=$ television, $\mathrm{X} 2=$ printed media, $\mathrm{X} 3=$ mobile phones, $\mathrm{X} 4=$ health facilities, $\mathrm{X} 5=$ radios, $\mathrm{X} 6=$ age group of the women, $X 7=$ place of residence of the women, $X 8=$ education level of the women, $X 9=$ wealth index of the women, and $\mathrm{X} 10=$ marital status of the women; $\varepsilon=$ error term which means all variables (factors) which affected use of FP but are not considered in this study. Results (Table 4) also show that printed media predicted more on the likelihood on the use of FP at $\beta=0.460$ and $\mathrm{p}<0.001$; followed by mobile phone with $\beta=0.392$ and $p<0.056$; radio at $\beta=0.368$ and $p<0.001$; health facilities $\beta=0.284$ and $p<0.001$; education level with $\beta=0.276$ and $p<0.001$, television with $\beta=0.168$ and $p<0.050$ and, age group of the women with $\beta=-0.035$ and $p<0.001$. Area of residence, wealth index and marital status were not statistically significant at $\mathrm{p}<0.419, \mathrm{p}<0.146, \mathrm{p}<0.609$ respectively. Along with this view, Nettey et al. (2016) point that market interaction among women, water fetching interaction, games and hairdressing salon are growing sources of FP messages dissemination.

\section{Discussion}

Frequency of Access to FP Messages and the Use of FP: Results in this study indicate that the percentage of the women who used FP services increased with the increased fluency of access to FP messages. Variation in use of FP among women who have occasionally received FP messages and who frequently received FP messages was observed. These findings are in line with those by Tisha et al. (2015) study in Pakistan that revealed that media exposure influenced FP use. The study also indicated that women who used FP are those who were exposed to any sort of media. Various studies in several countries, including, but not limited to, Bangladesh, Nigeria, Nepal, and Tanzania, have demonstrated similar results which support the positive association between exposure to FP mass media campaigns and FP use, intention, and/or knowledge (Hutchinson \& Wheeler, 2006 and Guilkey \& Hutchinson, 2011 in Jacobs, 2016). Although the authors considered them as relevant sources for FP message arguably, they are most likely associated with negative impacts on the use of FP as messages are not provided by health professionals.

Spatial, Socio-Demographic Characteristics of the Women' and Access to Sources of FP Messages: The study findings show that there is a statically significant correlation between all of the independent variables and access to FP messages via radios, television, printed media and mobile phones. The study findings further revealed that access to FP messages via health facilities had a statically significant correlation with marital status, education, place of residence and wealth index of the women, while access to FP messages via field workers was not. Of the communication media, radio, health facilities, television, printed media, field workers and mobile phone were the most used sources of FP messages.

Access to FP Sources and Spatial and Socio-Demographic and the Use of FP: The study found that the use of printed media was more likely to predict women's likelihood on the use of FP than mobile phones. The study also found that access to FP messages via health facilities predict women likelihood to use of FP. These findings are similar to a study by (Ajala, 2015) in Nigeria which revealed that women who are exposed to print media report intent to use any form of FP. The study in Kenya on the effectiveness of FP messages via mobile phone also revealed that most FP users who self-report behaviour changes, including contraceptive use are of the young age (Apanga \& Adam, 2015). The study by Basten (2010) has also found that FP messages via radio significantly influence listeners' awareness of FP, as they help to improve their attitude towards fidelity and family relations, as well as to adopt FP techniques. Similarly, in Tanzania, a radio soap opera entitled Tendon Waikato was deemed to have had 'strong behavioural effects on FP adoption; it increased listeners' self-efficacy regarding FP adoption and influenced listeners to talk to their spouses and 
peers about contraception' (Vaughan \& Rogers, 2000 cited by Basten, 2010). The findings on the access to FP messages via health facilities and their influence on women's likelihood to use FP coincide with the findings by Odewale (2016) which revealed that married women who heard FP information at health facilities were 1.5 times as likely to report the use of contraception as those who did not. The result has shown that women's level of education was associated with their likelihood to use FP.

These findings are consistent with the findings by Gaetano et al. (2014) and Apanga \& Adam (2015) which found that educated women are more likely to use FP services as compared to their peers who do not receive a formal education. Women's education significantly influences their approval of FP and increased their positive attitudes towards modern FP (Oye-Adeniran et al., 2006 cited by Ejembi et al., 2015; Islam \& Hasan, 2016). Women who have access to television are about ten times more likely to use FP compared to those who did not (Anguko, 2014). Similar findings are reported in Pakistan, India and Bangladesh (Apanga \& Adam, 2015).

\section{Conclusions and Recommendations}

The paper set to examine the relationship between accesses to communication channels on the use of FP among women aged 14-49 years; how the exposure to FP messages and women's demographic characteristics affect the use of FP. The findings have revealed that there is a statistical significant correlation between access to FP messages and the use of FP. Access to FP messages via radio, television, printed media, and mobile phones is observed more among women who are not in a union, those with a tertiary level of education, those in urban areas, and those within the highest wealth index. Printed media has a higher prediction on the women likelihood on the use of FP, followed by mobile phone, radio, health facilities, and level of education level. We propose that the Tanzanian government and development partners need to promote an intensified program for dissemination of FP messages, and more preferably through a combination of different channels. Education to girls cannot be overstated as it will not only help them to increase their economic status, but also it will enhance their decision relating to FP. Finally, there is a need for further research on the determinants of the selection of FP methods among women in the country.

Policy Implications: As access to FP messages, women's level of education and wealth status are positively associated with FP use, it is evident that investment on the use of integrated FP communications channels: radio, television, mobile phone and health facilities, as well as women's economic empowerment, should command the attention of the government, development partners and development policy-makers in Tanzania. To this end, greater investment on the education of girls should be pursued, as it has multiplier effects in increasing uptake of FP methods.

Authors' Contributions: Zuena Kilugwe and Sosthenes Ruheza conceptualized the research idea. The Authors both analysed the data, and wrote the draft of the manuscript. The final manuscript was read, edited and approved by both Authors.

Acknowledgements: The researchers are grateful to the Tanzania Demographic Health Survey and Malaria Indicator Survey of 2015-2016 for giving the permission to access the TDHS 2015 data set from the measure website.

\section{Reference}

Ajaero, C., Odimegwu, C., Ajaero, I. \& Nwachukwu, C. (2016). Access to mass media messages, and use of family planning in Nigeria: a spatio-demographic analysis from the 2013 DHS. BMC Public Health, 16, 427.

Ajala, A. (2015). Mass Media Exposure and Intention to use Contraceptives in North-west Geo-political Zone, Nigeria. Developing Countries Studies, 4(24).

Amin, T. (2014). Trend and Pattern of Use and Barriers to Family Planning in Egypt. International Public Health Forum, 1(4). 
Anguko, A. (2014). A thesis Submitted to the University of Nairobi, Institute of Tropical Infectious Diseases (UNITID) for Partial Fulfilment of the Requirement for the Degree of Master of Sciences in Medical Statistics, 50.

Apanga, W. A. (2015). Factors Influencing the Uptake of Family Planning Services in the Talensi District, Ghana. The Pan African Medical Journa, 2015.

Basten, S. (2010). Television and Fertility. Finnish Yearbook of Population Research XLV 2010, 67-82.

Gaetano, M., Lutuf, A., Zaake, D. \& Annika, J. (2014). Predictors of Contraceptive use Among Female Adolescents in Ghana 102. African Journal of Reproductive Health, 18(1), 102.

Grant, C. \& Bhardwaj, M. (2016). Family Planning Communications. K4D Helpdesk Research Report, Institute of Development Studies., Brighton, UK.

Gupta, N., Katende, C. \& Bessinger, R. (2003). Association of mass media exposure on family planning attitudes and practices in Uganda. . MEASURE Evaluation Working Papers.

Hamid, S. \& Stephenson, R. (2006). Provider and Health Facility Influences on Contraceptive Adoption in Urban Pakistan. International Family Planning Perspectives, 32, 71-78.

International Planned Parenthood Federation (IPPF). (2013). Family Planning. International conference of family planning. Addis Ababa-Ethiopia: IPPF https://www.ippf.org/family-planning-addis.

Islam, S. \& Hasan, M. (2016). Women Knowledge, Attitude, Approval of Family Planning and Contraceptive Use in Bangladesh. Asia Pacific Journal of Multidisciplinary Research, 4(2), 76-82.

Jacobs, J. (2016). Mass Media Exposure and Family Planning in West African Adolescents. Scholar Archive, Paper 3760.

Khan, T. \& Khan, R. (2010). Fertility Behaviours of Women and their Household Characteristics: a case of Punjab, Pakistan. Journal of Human Ecology, 30(1), 11-17.

Longwe, A., Huisman, J. \& Smits, J. (2012). Effects of knowledge, acceptance and use of contraceptives on household wealth in 26 African Countries. Nijmegen Center for Economics (NiCE). Radboud University. Institute for Management Research .

Miller, A. (2011). The effect of motherhood timing on career path. Journal of Population Economics, 24, 10711100.

Ministry of Health, Community Development, Gender, Elderly and Children (MoHCDGEC) [Tanzania Mainland], Ministry of Health (MoH) [Zanzibar], National Bureau of Statistics (NBS), Office of the Chief Government Statistician (OCGS), and ICF. 2016. Tanzania Demographic and Health Survey and Malaria Indicator Survey (TDHS-MIS) 2015-16. Dar es Salaam, Tanzania, and Rockville, Maryland, USA:MoHCDGEC, MoH, NBS, OCGS, and ICF.

National Bureau of Statistics (NBS) Tanzania. (2015). Population Projections. Dar es Salaam,Tanzania: NBS.

Nettey, O., Yeetey, E. A., Mahama, E., Sulemana, A., Adjei, G. \& Gyaase, S. (2015). Family Planning Awareness, Perceptions and Practice among Community Members in the Kintampo Districts of Ghana. Advances in Reproductive Sciences, 3, 1-12.

Ochako, R., Mbondo, M., Aloo, S., Kaimenyi, S., Thompson, R. \& Temmerman, M. (2015). Barriers to Modern Contraceptive Methods Uptake among Young Women in Kenya: a Qualitative Study. BMC Public Health, 15, 118.

Odewale, B. (2016). Multiple Exposure to Information about Family Planning and Contraceptive Use among Women in Nigeria. 3rd International Conference on African Development Issues. (CU-ICADI 2016) , (pp. 573-576).

Oseko, B. (2013). The role of Interpersonal Communication in the use of Family Planning Methods Among Women. A study of Kajiado County. A research project submitted in Partial Fulfilment for the Requirements of the Award of Masters Degree in Communication Studies at the University of Nairobi. Pp 60.

Tisha, S., Haque, S. M. \& Tabassum, M. (2015). Antenatal Care, an Expediter for Postpartum Modern Contraceptive Use. Research in Obstetrics and Gynecology, 3(2), 22-31.

Westoff, C. (2012). The recent fertility transition in Rwanda. Population and Development Review 389 Supplement, 169-178. 